Author affiliations and support information (if applicable) appear at the end of this article.

Published at jco.org on August 29, 2018. Corresponding author: Adam C. Calaway, $\mathrm{MD}$, Department of Urology, Indiana University School of Medicine, $535 \mathrm{~N}$ Barnhill Dr, Suite 150, Indianapolis, IN 46202; Twitter: @IUuro, @AdamCalaway; e-mail: calawaya@iupui.edu.

C 2018 by American Society of Clinical Oncology

0732-183X/18/3629w-2950w/\$20.00
DOI: https://doi.org/10.1200/JCO 18.00431

\title{
Risk of Bleomycin-Related Pulmonary Toxicities and Operative Morbidity After Postchemotherapy Retroperitoneal Lymph Node Dissection in Patients With Good-Risk Germ Cell Tumors
}

Adam C. Calaway, Richard S. Foster, Nabil Adra, Timothy A. Masterson, Costa Albany, Nassar H. Hanna, Lawrence H. Einhorn, and Clint Cary

\section{$\begin{array}{llllllll}\text { A } & \text { B } & \mathbf{S} & \mathbf{T} & \mathbf{R} & \mathbf{A} & \mathbf{C} & \mathbf{T}\end{array}$}

\section{Purpose}

Three cycles of bleomycin, etoposide, and cisplatin (BEP $\times 3$ ) or four cycles of etoposide and cisplatin $(E P \times 4)$ are first-line chemotherapy regimens for men with International Germ Cell Cancer Collaborative Group (IGCCCG) good-risk germ cell tumors (GCTs). We determined whether inclusion of bleomycin affected pulmonary and operative morbidity after postchemotherapy retroperitoneal lymph node dissection (PC-RPLND).

\section{Patients and Methods}

We queried our database to identify IGCCCG good-risk patients who received BEP $\times 3$ or EP $\times 4$ induction chemotherapy before PC-RPLND from 2006 to 2016. Patients who received combination regimens were excluded. The primary outcomes of interest were pulmonary morbidity (prolonged intubation, reintubation, supplemental oxygen use, intensive care unit stay) and operative morbidity (operative time, length of stay, concomitant procedures, estimated blood loss).

\section{Results}

We analyzed 234 patients (191 BEP $\times 3$ v43 EP $\times 4$ ). All patients were extubated immediately after the operation. None were reintubated or discharged on oxygen. Two patients in each cohort required an intensive care unit stay for nonpulmonary reasons. Patients treated with BEP required shorter use of supplemental oxygen (0.99 $\vee 1.63$ days; $P=.005)$. No significant differences were found in preoperative mass size $(P=.42)$ or concomitant surgeries $(P=.58)$. Operative time was significantly shorter (131 v170 minutes; $P<.01$ ), and estimated blood loss was considerably less (194 v226 mL; $P<.01)$ in patients treated with BEP. Length of stay was shorter in patients treated with BEP (3.3 $\mathrm{v}$ 3.9 days; $P<.01)$.

\section{Conclusion}

In a modern surgical cohort, the inclusion of bleomycin does not seem to influence pulmonary morbidity, operative difficulty, or nonpulmonary postoperative complications after PC-RPLND in men with IGCCCG good-risk GST.

\section{J Clin Oncol 36:2950-2954. (C) 2018 by American Society of Clinical Oncology}

\section{INTRODUCTION}

The discovery of the unique sensitivity of nonseminomatous germ cell tumors (GCTs) to cisplatin-based combination chemotherapy regimens revolutionized the treatment and survival of men with testicular cancer. ${ }^{1}$ Initial regimens of cisplatin, vinblastine, and bleomycin yielded complete response rates of $74 \%$ and long-term survival rates of $70 \%$, but treatment toxicity was high. ${ }^{2-4}$ As a result, the goals of treatment shifted to identify induction chemotherapy regimens that limited treatment-related toxicities while maintaining excellent cure rates, especially in men with good-risk disease.

Numerous randomized controlled trials investigated various induction regimens. ${ }^{4-11}$ Currently, the National Comprehensive Cancer Network endorses either three cycles of bleomycin, etoposide, and cisplatin $(\mathrm{BEP} \times 3)$ or four cycles of etoposide and cisplatin $(\mathrm{EP} \times 4)$ as acceptable induction chemotherapy regimens for patients with International Germ Cell Cancer 
Collaborative Group (IGCCCG) good-risk disease. ${ }^{12}$ However, the debate continues about which chemotherapy regimen is best for men with good-risk disease.

A recent publication from our group evaluated the risk of postoperative complications in patients who undergo resection of primary mediastinal GCTs after chemotherapy. ${ }^{13}$ Complications were higher in men who received BEP compared with etoposide, ifosfamide, and cisplatin, likely because of extensive chest surgery; thus, these findings prompted us to determine whether the same was true with abdominal surgery. Therefore, in the current study, we investigated the incidence and significance of bleomycin-related pulmonary and operative morbidity in men with IGCCCG goodrisk nonseminomatous GCTs who underwent postchemotherapy retroperitoneal lymph node dissection (PC-RPLND). We hypothesized that the inclusion of bleomycin in induction regimens would not increase pulmonary or operative morbidity, which may alleviate some of the toxicity concerns of this regimen.

\section{PATIENTS AND METHODS}

\section{Study Cohort}

Our prospectively maintained Indiana University Testis Database was queried to identify IGCCCG good-risk patients who underwent PCRPLND between 2006 and 2016 and received either BEP $\times 3$ or EP $\times 4$. Inclusion criteria were men diagnosed with nonseminomatous GCTs of the testis, good-risk disease as defined by IGCCCG criteria, receipt of either $\mathrm{BEP} \times 3$ or EP $\times 4$ as induction chemotherapy, residual retroperitoneal mass $>1 \mathrm{~cm}$, and normal preoperative serum tumor markers. We began the study period in 2006 because intraoperative anesthesia records had become readily retrievable. A total of 234 patients met criteria for evaluation; 191 received $\mathrm{BEP} \times 3$, and 43 received $\mathrm{EP} \times 4$.

\section{Anesthetic Management}

The anesthetic management is similar at our institution for all patients who undergo RPLND for residual disease regardless of induction chemotherapy regimen. Brief episodes of a high fraction of inspired oxygen $\left(\mathrm{FiO}_{2}\right)$ are used during induction of and emergence from anesthesia. The $\mathrm{FIO}_{2}$ is substantially decreased for the remainder of the operation. Typically, the $\mathrm{FIO}_{2}$ is kept between $30 \%$ and $40 \%$ for the majority of the operation. Intraoperative resuscitation is minimized and not driven by intraoperative urine output. Additional fluid is not given to compensate for nothing by mouth status. Patients typically are given $4 \mathrm{~mL} / \mathrm{kg} / \mathrm{h}$ lactated ringers and $2 \mathrm{~mL} / \mathrm{kg} / \mathrm{h}$ albumin for the first 2 hours of the operation. Volume and vital status are then reassessed at that point to dictate additional resuscitation. Blood transfusions are administered only if clinically indicated. After the completion of the operation and before emergence, patients typically are placed in lateral decubitus position and administered a single shot of intrathecal narcotic.

\section{Independent Variables and Outcome}

The primary outcomes of the study were pulmonary and operative morbidity. Pulmonary outcomes included prolonged intubation (defined as $>1$ day), need for reintubation, intensive care unit need and duration, and supplemental oxygen duration. Operative outcomes included operative time, length of stay, estimated blood loss, and performance of concomitant procedures.

\section{Statistical Analysis}

Baseline characteristics of the BEP $\times 3$ and $\mathrm{EP} \times 4$ groups were compared using $t$ tests for differences in means of normally distributed continuous variables and the Mann-Whitney $U$ test for nonnormally
Table 1. Preoperative Demographics and Clinical Data on the Basis of Induction Chemotherapy Regimen

\begin{tabular}{|c|c|c|c|}
\hline Characteristic & $\begin{array}{c}\text { BEP Cohort, } \\
\text { No. }(\%)\end{array}$ & $\begin{array}{c}\text { EP Cohort, } \\
\text { No. }(\%)\end{array}$ & $P$ \\
\hline No. of patients & 191 & 43 & \\
\hline Age, years (IQR) & $27(22-32)$ & $31(25-41)$ & $<.01$ \\
\hline Orchiectomy pathology & & & .33 \\
\hline NSGCT & $176(92)$ & 37 (79) & \\
\hline Seminoma* & $2(1)$ & $0(0)$ & \\
\hline Othert & $9(5)$ & $4(9)$ & \\
\hline Missing & $4(2)$ & $2(5)$ & \\
\hline Clinical stage at presentation & & & .12 \\
\hline । & $65(34)$ & $7(16)$ & \\
\hline$\|$ & $81(42)$ & $23(54)$ & \\
\hline III & $28(15)$ & $9(21)$ & \\
\hline Missing & $17(9)$ & $4(9)$ & \\
\hline Preoperative mass size, $\mathrm{cm}$ & & & .42 \\
\hline$<2$ & 27 (19) & $8(21)$ & \\
\hline $2-5$ & $80(57)$ & $18(46)$ & \\
\hline$>5$ & $34(24)$ & $13(33)$ & \\
\hline Missing & 50 & 4 & \\
\hline
\end{tabular}

Abbreviations: BEP, bleomycin, etoposide, and cisplatin; EP, etoposide and cisplatin; IQR, interquartile range; NSGCT, nonseminomatous germ cell tumor.

*Both patients with seminoma had elevated alpha-fetoprotein at presentation. tOther consists of scar, intratubular germ cell neoplasia, and fibrosis.

distributed continuous variables. Pearson $\chi^{2}$ and Fisher's exact tests were used for categorical variables. All statistical tests were two sided with significance set at $P<.05$. Stata/IC 12.1 statistical software (StataCorp, College Station, TX) was used to conduct the analysis.

\section{RESULTS}

Table 1 lists the baseline demographic and clinical characteristics of the study cohort stratified on the basis of induction chemotherapy regimen. The group characteristics were similar. Men who received $\mathrm{BEP} \times 3$ were younger than those who received $\mathrm{EP} \times 4(27 v 31$ years; $P<.01)$. Indications for the use of $\mathrm{EP} \times 4$ were referring oncologist preference $(28[65.1 \%])$, patient age older than 50 years (seven [16.2\%]), pulmonary insufficiency at baseline (two [4.7\%]), and extensive pulmonary metastatic disease at presentation (six $[14 \%])$. None of these patients were treated or seen before the start of chemotherapy at our institution. Clinical stage at presentation was similar between the groups $(P=.12)$. No significant differences were seen in preoperative mass size between groups $(P=.42)$.

Pulmonary morbidity after PC-RPLND was low in all patients regardless of induction chemotherapy regimen. All patients were successfully extubated at the conclusion of the operation, and no patient in either group required postoperative reintubation. Only four patients required postoperative monitoring in the intensive care unit, all for nonpulmonary-related issues (two of whom received BEP $\times 3$ and two of whom received $\mathrm{EP} \times 4$ ). The duration of supplemental oxygen use was shorter in patients treated with $\mathrm{BEP} \times 3(0.98 v 1.61$ days; $P<.01)$. Pulmonary-related outcomes on the basis of chemotherapy type are listed in Table 2. These outcomes occurred despite concomitant thoracic procedures being more common in patients who underwent $\mathrm{BEP} \times 3$ versus $\mathrm{EP} \times 4$ (Table 3 ). Briefly, seven patients who underwent $\mathrm{BEP} \times 3$ required thoracic procedures. Two patients underwent video-assisted 


\begin{tabular}{|lccc}
\hline $\begin{array}{l}\text { Table 2. Pulmonary Morbidity After Postchemotherapy Retroperitoneal Lymph } \\
\text { Node Dissection on the Basis of Induction Chemotherapy Regimen }\end{array}$ \\
\hline \multicolumn{1}{c}{ Variable } & $\begin{array}{c}\text { BEP Cohort } \\
(\mathrm{n}=191)\end{array}$ & $\begin{array}{c}\text { EP Cohort } \\
(\mathrm{n}=43)\end{array}$ & $P$ \\
\hline $\begin{array}{l}\text { Intubation duration, days } \\
\text { No. needing reintubation }\end{array}$ & 0 & 0 & $\mathrm{NA}$ \\
$\begin{array}{l}\text { No. needing intensive care unit } \\
\text { stay (\%) }\end{array}$ & 0 & 0 & $\mathrm{NA}$ \\
$\begin{array}{l}\text { Mean duration of supplemental } \\
\text { oxygen, days }\end{array}$ & 0.99 & $2(5)$ & .054 \\
\hline
\end{tabular}

Abbreviations: BEP, bleomycin, etoposide, and cisplatin; EP, etoposide and cisplatin; NA, not applicable.

thoracotomies for small-volume residual parenchymal lesions. Five patients underwent open thoracotomy during which two required only wedge resections (eight lesions total), and three required a mediastinal dissection and/or lobectomy. The two patients who received $\mathrm{EP} \times 4$ and underwent concomitant thoracic procedures had higher-volume disease in the chest at the time of diagnosis. One patient required a sternotomy, thymectomy, anterior superior mediastinal dissection, and removal of a large paratracheal residual mass. The other patient required a thoracotomy, extensive mediastinal dissection, and a pulmonary wedge resection.

Operative morbidity after PC-RPLND was similarly low in all patients, although a few differences were seen between groups. Operative time was approximately 40 minutes shorter for those who received $\mathrm{BEP} \times 3(131 v 170$ minutes; $P<.01)$. Estimated blood loss also was lower in the BEP $\times 3$ cohort $(194 v 226 \mathrm{~mL}$; $P<.01)$ as was length of stay ( $3.3 v 3.9$ days; $P<.01)$. Only five patients required a blood transfusion during the operation or the postoperative stay (three $\mathrm{BEP} \times 3 v$ two $\mathrm{EP} \times 4$ ). These differences in operative outcomes between the two groups were not a result of differences in preoperative mass size $(P=.42)$ or concomitant procedures $(8.9 \% v 11.6 \%$; $P=.58)$. Operative outcomes on the basis of chemotherapy type are listed in Table 3.

\section{DISCUSSION}

The current study demonstrates that pulmonary toxicity and operative morbidity at the time of PC-RPLND in men with IGCCCG good-risk nonseminomatous GCTs was exceedingly low in those who received bleomycin as a component of the induction chemotherapy regimen. No patient required prolonged intubation or reintubation. All patients, regardless of regimen, required supplemental oxygen for only 1 postoperative day. In addition, estimated blood loss and operative times statistically favored $\mathrm{BEP} \times 3$, but the clinical significance of this is uncertain. Finally, the proportion of patients who required additional procedures at the time of PC-RPLND were similar between groups.

The concern for bleomycin-induced pulmonary toxicity $(\mathrm{BPT})$ is one of the most common reasons why $\mathrm{EP} \times 4$ is preferred over BEP $\times 3$ in men with IGCCCG good-risk metastatic GCTs. However, studies that evaluated the risk of BPT in this setting do not support such concerns. A prospective cohort study of 835 men treated with bleomycin-containing chemotherapy regimens assessed the incidence and risk factors of BPT. Overall, only $6.8 \%$ of patients had BPT, and the risk of death directly attributable to BPT was $1 \%$. Factors that independently predict BPT were renal function (glomerular filtration rate, $<80 \mathrm{~mL} / \mathrm{min}$ ), age ( $>40$ years), and cumulative dose of bleomycin ( $>300 \mathrm{IU}$ ). Rates of BPT using BEP $\times 3$ (30 IU weekly $\times 9=270 \mathrm{IU}$ ) for good-risk disease were much lower than rates of BPT for men with intermediate- to high-risk disease administered $\mathrm{BEP} \times 4(30 \mathrm{IU}$ weekly $\times 12$ weeks $=360 \mathrm{IU}) .{ }^{14}$ These results were corroborated by a prospective, unselected (ie, containing men with all IGCCCG risk groups) cohort study. Acute and chronic pulmonary complications in 565 men treated with BEP for metastatic GCTs were minimal and predominantly limited to high-risk individuals (IGCCCG poor risk, pulmonary surgery, and pulmonary embolus before or during treatment). ${ }^{15}$ Finally, in a prospective randomized trial that compared the efficacy and toxicity of BEP $\times 3$ versus $\mathrm{EP} \times 4$ in good-risk nonseminomatous GCTs, pulmonary toxicity occurred in 12 patients who received BEP $\times 3$ and eight patients who received $\mathrm{EP} \times 4(P=.38){ }^{16}$

A meta-analysis of randomized controlled trials that evaluated the incidence of BPT in men with metastatic GCTs published contrasting results. The probability of all-grade and high-grade pulmonary toxicity was higher in men treated with bleomycin than in those who were not ( $12 \% v 2 \%$ and $6 \% v 1 \%$, respectively). On multivariable analysis, bleomycin increased the odds of all-grade (but not high-grade) pulmonary toxicity. ${ }^{17}$ This study included men treated with various chemotherapy dosages and regimens and IGCCCG risk categories. When specifically evaluating men with IGCCCG good-risk disease treated with 270 IU bleomycin, the event rate of all-grade and high-grade pulmonary toxicity is exceeding low, with some large trials reporting a $0 \%$ incidence of $\mathrm{BPT}^{7,17}$ Thus, when evaluating the totality of the existing evidence, $\mathrm{BPT}$ is extremely rare in patients treated with $\mathrm{BEP} \times 3$. The current study in a surgical cohort supports this statement and adds that peri-operative pulmonary complications are minimal in men who require a PC-RPLND for residual disease, regardless of induction chemotherapy regimen. In fact, no patient in either chemotherapy group experienced a significant pulmonary complication during the peri-operative or immediate postoperative period. This contrasts our group's previous findings that bleomycin-containing chemotherapy compared with etoposide, ifosfamide, and cisplatin greatly increases postoperative pulmonary complications in men

\begin{tabular}{|c|c|c|c|}
\hline Variable & $\begin{array}{l}\text { BEP Cohort } \\
(\mathrm{n}=191)\end{array}$ & $\begin{array}{l}\text { EP Cohort } \\
(n=43)\end{array}$ & $P$ \\
\hline Operative time, minutes & 131 & 170 & $<.01$ \\
\hline Estimated blood loss, $\mathrm{mL}$ & 194 & 243 & .01 \\
\hline Length of stay, days & 3.3 & 3.9 & $<.01$ \\
\hline $\begin{array}{l}\text { No. of additional procedures } \\
\text { at time of RPLND (\%) }\end{array}$ & $17(8.9)$ & 5 (11.6) & .58 \\
\hline Nephrectomy & 1 & 1 & \\
\hline Thoracic procedure & 7 & 2 & \\
\hline Vascular procedure & 3 & 0 & \\
\hline Bowel resection & 2 & 2 & \\
\hline Orchiectomy & 1 & 0 & \\
\hline $\begin{array}{l}\text { Appendectomy/Meckel's } \\
\text { diverticulectomy }\end{array}$ & 2 & 0 & \\
\hline Ureteral resection & 1 & 0 & \\
\hline
\end{tabular}

Abbreviations: BEP, bleomycin, etoposide, and cisplatin; EP, etoposide and cisplatin; RPLND, retroperitoneal lymph node dissection. 
who require extensive chest surgery for primary mediastinal GCT. ${ }^{13}$ Thus, selection of induction chemotherapy regimen in men with IGCCCG good-risk GCTs with minimal pulmonary disease should be made independently of the concern for pulmonary toxicity, except in men with documented risk factors (age, poor renal function, preexisting pulmonary insufficiency).

Complication rates after PC-RPLND are substantially higher than after primary RPLND. A retrospective study that evaluated 1,081 men who underwent PC-RPLND or primary RPLND reported major complication rates of $18 \%$ and $8 \%$, respectively. ${ }^{18,19}$ Obvious differences in the clinical scenario explain the differences in complication rates, including stage of disease, size of lymph node mass, and presence of postchemotherapy desmoplastic reaction. Some have hypothesized that bleomycin-containing chemotherapy regimens increase surgical morbidity. ${ }^{20}$ The current study does not substantiate such claims. Operative morbidity was low in all patients in the current study, regardless of type of induction chemotherapy, which suggests that chemotherapy regimen, especially those that contain bleomycin, does not influence operative morbidity and does not have an effect on the surgical dissection.

The current study should be considered in the context of certain limitations. First, the inherit weakness of a retrospective analysis of a prospectively maintained database are well documented. However, no previous clinical trial or prospective cohort to our knowledge has reported pulmonary and operative morbidity stratified by chemotherapy regimen in men with IGCCCG goodrisk disease. Second, our institutional preference of using BEP induction chemotherapy in men younger than 50 years old without significant pulmonary comorbidities is well known, which may represent inherent selection bias. However, as a tertiary referral center, patients often present with residual retroperitoneal disease after the completion of chemotherapy; therefore, the induction chemotherapy regimen often is at the discretion of the referring oncologist. This is highlighted by the fact that only 30 patients $(12.8 \%)$ in the current cohort were treated or seen at our institution before the start of chemotherapy. Third, pulmonary morbidity was not assessed using the results of pulmonary function tests (PFTs). We instead opted to evaluate this end point by using clinically important variables, such as need for prolonged intubation and supplemental oxygen use. Of note, we routinely do not perform PFTs for good-risk patients who receive $\mathrm{BEP} \times 3$ unless they have an abnormal history or physical examination. The only patients in whom we occasionally perform PFTs are those with intermediate- or poor-risk disease. In these patients, PFTs sometimes are done at baseline and just before the fourth cycle to ensure that the 10th, 11th, and 12th doses of bleomycin can be administered safely. Fourth, because a majority of patients received chemotherapy at referring centers, dosing, dose reductions, and treatment delays were not reliably captured. However, we have previously demonstrated that good-risk patients who were treated with $\mathrm{BEP} \times 3$ at Indiana University versus an outside referral center and underwent PC-RPLND at our center had similar survival and histologic outcomes. ${ }^{21}$ This likely underscores the importance of surgical management at experienced referral centers for men with bulky and aggressive disease and of appropriate anesthetic and postoperative treatment of these individuals (lower $\mathrm{FIO}_{2}$, removal of supplemental oxygen quickly, etc).

These limitations notwithstanding, we believe that the data presented in this study add sufficient and compelling evidence about the safety of bleomycin in chemotherapy regimens for men with IGCCCG good-risk disease who require PC-RPLND. Pulmonary morbidity was low regardless of induction chemotherapy approach; therefore, the perceived pulmonary toxicity of bleomycin in this patient cohort is unsubstantiated. Although the improved operative outcomes may be linked to residual confounding and poorly explained clinically, it is safe to assume that operative difficulty and postoperative complications are not associated with chemotherapy regimen and are linked more strongly to surgeon experience, location of treatment, extent of disease, and preexisting medical comorbidities.

In conclusion, we found that pulmonary morbidity is not increased in men who receive bleomycin-containing chemotherapy for IGCCCG good-risk testicular cancer. Operative outcomes were similar, if not statistically improved, in the cohort that received BEP. This phenomenon is hard to explain yet justifies that the inclusion of bleomycin likely does not influence operative difficulty or nonpulmonary postoperative complications. Given these findings, oncologists should consider the indications of using EP regimens in this patient population and potentially limiting the use in older men with preexisting pulmonary disease.

\section{AUTHORS' DISCLOSURES OF POTENTIAL CONFLICTS OF INTEREST}

Disclosures provided by the authors are available with this article at jco.org.

\section{AUTHOR CONTRIBUTIONS}

Conception and design: Adam C. Calaway, Richard S. Foster, Timothy A. Masterson, Costa Albany, Nassar H. Hanna, Lawrence H. Einhorn, Clint Cary

Provision of study materials or patients: Lawrence H. Einhorn

Collection and assembly of data: Adam C. Calaway, Timothy A. Masterson, Costa Albany, Lawrence H. Einhorn, Clint Cary

Data analysis and interpretation: All authors

Manuscript writing: All authors

Final approval of manuscript: All authors

Accountable for all aspects of the work: All authors

\section{REFERENCES}

1. Einhorn LH, Donohue J: Cisdiamminedichloroplatinum, vinblastine, and bleomycin combination chemotherapy in disseminated testicular cancer. Ann Intern Med 87:293-298, 1977
2. Einhorn LH: Treatment of testicular cancer: A new and improved model. J Clin Oncol 8:1777-1781, 1990

3. Beck SDW, Foster RS, Bihrle R, et al: Outcome analysis for patients with elevated serum tumor markers at postchemotherapy retroperitoneal lymph node dissection. J Clin Oncol 23:6149-6156, 2005
4. Williams SD, Birch R, Einhorn LH, et al: Treatment of disseminated germ-cell tumors with cisplatin, bleomycin, and either vinblastine or etoposide. N Engl J Med 316:1435-1440, 1987

5. Levi JA, Raghavan D, Harvey V, et al: The importance of bleomycin in combination chemotherapy 
for good-prognosis germ cell carcinoma. J Clin Oncol 11:1300-1305, 1993

6. Bosl GJ, Geller NL, Bajorin D, et al: A randomized trial of etoposide + cisplatin versus vinblastine + bleomycin + cisplatin + cyclophosphamide + dactinomycin in patients with good-prognosis germ cell tumors. J Clin Oncol 6:1231-1238, 1988

7. Loehrer PJ Sr, Johnson D, Elson P, et al: Importance of bleomycin in favorable-prognosis disseminated germ cell tumors: An Eastern Cooperative Oncology Group trial. J Clin Oncol 13:470-476, 1995

8. de Wit R, Roberts JT, Wilkinson PM, et al: Equivalence of three or four cycles of bleomycin, etoposide, and cisplatin chemotherapy and of a 3- or 5-day schedule in good-prognosis germ cell cancer: A randomized study of the European Organization for Research and Treatment of Cancer Genitourinary Tract Cancer Cooperative Group and the Medical Research Council. J Clin Oncol 19:1629-1640, 2001

9. de Wit R, Stoter G, Kaye SB, et al: Importance of bleomycin in combination chemotherapy for goodprognosis testicular nonseminoma: A randomized study of the European Organization for Research and Treatment of Cancer Genitourinary Tract Cancer Cooperative Group. J Clin Oncol 15:1837-1843, 1997

10. Einhorn LH, Williams SD, Loehrer PJ, et al: Evaluation of optimal duration of chemotherapy in favorable-prognosis disseminated germ cell tumors: A Southeastern Cancer Study Group protocol. J Clin Oncol 7:387-391, 1989

11. Bajorin DF, Sarosdy MF, Pfister DG, et al: Randomized trial of etoposide and cisplatin versus etoposide and carboplatin in patients with good-risk germ cell tumors: A multiinstitutional study. J Clin Oncol 11:598-606, 1993

12. International Germ Cell Cancer Collaborative Group: International germ cell consensus classification: A prognostic factor-based staging system for metastatic germ cell cancers. J Clin Oncol 15: 594-603, 1997

13. Ranganath $P$, Kesler KA, Einhorn LH: Perioperative morbidity and mortality associated with bleomycin in primary mediastinal nonseminomatous germ cell tumor. J Clin Oncol 34:4445-4446, 2016

14. O'Sullivan JM, Huddart RA, Norman AR, et al: Predicting the risk of bleomycin lung toxicity in patients with germ-cell tumours. Ann Oncol 14:91-96, 2003

15. Lauritsen J, Kier MGG, Bandak M, et al: Pulmonary function in patients with germ cell cancer treated with bleomycin, etoposide, and cisplatin. J Clin Oncol 34:1492-1499, 2016

16. Culine $S$, Kerbrat $P$, Kramar $A$, et al: Refining the optimal chemotherapy regimen for good-risk metastatic nonseminomatous germ-cell tumors: A randomized trial of the Genito-Urinary Group of the French Federation of Cancer Centers (GETUG T93BP). Ann Oncol 18:917-924, 2007

17. Necchi $A$, Miceli $R$, Oualla $K$, et al: Effect of bleomycin administration on the development of pulmonary toxicity in patients with metastatic germ cell tumors receiving first-line chemotherapy: A meta-analysis of randomized studies. Clin Genitourin Cancer 15:213-220.e5, 2017

18. Baniel J, Foster RS, Rowland RG, et al: Complications of post-chemotherapy retroperitoneal lymph node dissection. J Urol 153:976-980, 1995

19. Cary C, Masterson TA, Bihrle R, et al: Contemporary trends in postchemotherapy retroperitoneal lymph node dissection: Additional procedures and perioperative complications. Urol Oncol 33:389. e15-389.e21, 2015

20. Winter C, Raman JD, Sheinfeld J, et al: Retroperitoneal lymph node dissection after chemotherapy. BJU Int 104:1404-1412, 2009

21. Cary C, Jacob JM, Albany $C$, et al: Long-term survival of good-risk germ cell tumor patients after postchemotherapy retroperitoneal lymph node dissection: A comparison of BEP $\times 3$ vs. EP $\times 4$ and treating institution. Clin Genitourin Cancer 16:e307-e313, 2018

\section{Affiliations}

All authors: Indiana University School of Medicine, Indianapolis, IN.

Prior Presentation

Presented at the 18th Annual Meeting of the Society of Urologic Oncology, Washington, DC, November 29-December 1, 2017, and the 113th Annual Meeting of the American Urological Association, San Francisco, CA, May 18-21, 2018. 


\section{AUTHORS' DISCLOSURES OF POTENTIAL CONFLICTS OF INTEREST}

Risk of Bleomycin-Related Pulmonary Toxicities and Operative Morbidity After Postchemotherapy Retroperitoneal Lymph Node Dissection in Patients With Good-Risk Germ Cell Tumors

The following represents disclosure information provided by authors of this manuscript. All relationships are considered compensated. Relationships are self-held unless noted. I = Immediate Family Member, Inst = My Institution. Relationships may not relate to the subject matter of this manuscript. For more information about ASCO's conflict of interest policy, please refer to www.asco.org/rwc or ascopubs.org/jco/site/ifc.

\section{Adam C. Calaway}

No relationship to disclose

\section{Richard S. Foster}

No relationship to disclose

\section{Nabil Adra}

Research Funding: Merck (Inst)

Timothy A. Masterson

No relationship to disclose

\section{Costa Albany}

Stock and Other Ownership Interests: Advaxis

Honoraria: Sanofi

Consulting or Advisory Role: Seattle Genetics, AstraZeneca, MedImmune Speakers' Bureau: Bayer AG, Sanofi

Research Funding: Astex Pharmaceuticals (Inst), Merck (Inst), BristolMyers Squibb (Inst), Eli Lilly (Inst), Bayer AG (Inst)

Travel, Accommodations, Expenses: Sanofi

\section{Nassar H. Hanna}

Research Funding: Merck (Inst), Bristol-Myers Squibb (Inst), AstraZeneca (Inst), MedImmune (Inst), Genentech (Inst)

Lawrence H. Einhorn

Stock and Other Ownership Interests: Amgen, Biogen, Celgene Consulting or Advisory Role: Celgene

Clint Cary

No relationship to disclose 